\section{(6) OPEN ACCESS}

\title{
BCG-specific lgG-secreting peripheral plasmablasts as a potential biomarker of active tuberculosis in HIV negative and HIV positive patients
}

\author{
Senait Ashenafi, ${ }_{1}^{1,2}$ Getachew Aderaye, ${ }^{3}$ Martha Zewdie, ${ }^{4}$ Rubhana Raqib, ${ }^{5}$ \\ Amsalu Bekele, ${ }^{3}$ Isabelle Magalhaes, ${ }^{6}$ Beede Lema, ${ }^{7}$ Meseret Habtamu, ${ }^{4}$ \\ Rokeya Sultana Rekha, ${ }^{5}$ Getachew Aseffa, ${ }^{8}$ Markus Maeurer, ${ }^{6}$ Abraham Aseffa, ${ }^{4}$ \\ Mattias Svensson, ${ }^{1}$ Jan Andersson, ${ }^{1,9}$ Susanna Brighenti ${ }^{1}$
}

\begin{abstract}
- Additional supplementary files are published online only. To view these files please visit the journal online (http://dx. doi.org/10.1136/thoraxjnl2012-201817).
\end{abstract}

For numbered affiliations see end of article.

\section{Correspondence to} Dr Susanna Brighenti, Department of Medicine, Center for Infectious Medicine (CIM), Karolinska Institutet, Karolinska University Hospital Huddinge, 14186 Stockholm, Sweden;

susanna.brighenti@ki.se

Received 24 February 2012 Accepted 27 July 2012 Published Online First 24 August 2012

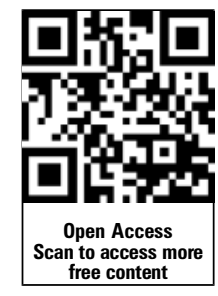

\section{Linked}

- http://dx.doi.org/10.1136/ thoraxjnl-2012-202481

To cite: Ashenafi S, Aderaye $\mathrm{G}$, Zewdie $\mathrm{M}$, et al. Thorax 2013;68:269-276.

\section{ABSTRACT}

Background Diagnosis of active tuberculosis (TB) among sputum-negative cases, patients with HIV infection and extra-pulmonary TB is difficult. In this study, assessment of BCG-specific lgG-secreting peripheral plasmablasts, was used to identify active TB in these high-risk groups.

Methods Peripheral blood mononuclear cells were isolated from patients with TB and controls and cultured in vitro using an assay called Antibodies in Lymphocyte Supernatant, which measures spontaneous IgG antibody release from migratory plasmablasts. A BCG-specific ELISA and flow cytometry were used to quantify in vivo activated plasmablasts in blood samples from Ethiopian subjects who were HIV negative or HIV positive. Patients diagnosed with different clinical forms of sputumnegative active TB or other diseases ( $n=96)$ were compared with asymptomatic individuals including latent TB and non-TB controls $(n=85)$. Immunodiagnosis of TB also included the tuberculin skin test and the interferon (IFN)- $\gamma$ release assay, QuantiFERON.

Results This study demonstrated that circulating $\lg \mathrm{G}+$ plasmablasts and spontaneous secretion of BCG-specific $\lg G$ antibodies were significantly higher in patients with active TB compared with latent TB cases and non-TB controls. BCG-specific lgG titres were particularly high among patients coinfected with TB and HIV with CD4 T-cell counts $<200$ cells $/ \mathrm{ml}$ who produced low levels of Mycobacterium tuberculosis-specific IFN $\gamma$ in vitro.

Conclusions These results suggest that BCG-specific IgG-secreting peripheral plasmablasts could be successfully used as a host-specific biomarker to improve diagnosis of active TB, particularly in people who are HIV positive, and facilitate administration of effective treatment to patients. Elevated $\mathrm{IgG}$ responses were associated with impaired peripheral T-cell responses, including reduced T-cell numbers and low $M$ tuberculosis-specific IFN $\gamma$ production.

\section{INTRODUCTION}

Tuberculosis (TB), caused by Mycobacterium tuberculosis (Mtb), is one of the most important global health problems. Diagnosis of TB is complex as there are different clinical forms with various symptoms of infection and disease, including

\section{Key messages}

What is the key question?

- Can detection of BCG-specific lgG-secreting plasmablasts in the peripheral blood be used as a biomarker of active tuberculosis (TB) in sputum-negative patients who are HIV negative or HIV positive?

\section{What is the bottom line?}

- In contrast to conventional serology, in vitro assessment of in vivo activated BCG-specific IgG-secreting plasmablasts could be used as a potential diagnostic biomarker for different clinical forms of active TB in patients who are sputum- and culture-negative, particularly in those coinfected with TB and HIV with low CD4 T-cell counts and an impaired in vitro interferon- $\gamma$ response.

\section{Why read on?}

- BCG-specific IgG-secreting plasmablasts could be a promising biomarker for the development and clinical implementation of a simple point-of-care test that may enhance administration of appropriate chemotherapy to patients with TB in whom a diagnosis is difficult. These findings are of potential clinical relevance and also contribute to understanding the immunobiology of TB and TB/HIV coinfection.

coinfections with other pathogens such as HIV. ${ }^{1}$ To date, methods used for TB diagnosis include assessment of clinical symptoms, pulmonary $\mathrm{x}$-ray, direct microscopy of sputum samples, Mtb culture, cytohistopathology, PCR and immunological techniques such as the tuberculin skin test (TST) and interferon (IFN)- $\gamma$ release assays (IGRAs), that is, QuantiFERON-TB Gold in-Tube (QFTG) and T-SPOT.TB. However, these methods have important limitations and are often slow, expensive and require advanced equipment or invasive procedures that are difficult to use routinely in resource-poor settings. About $50 \%$ of patients with culture- 
confirmed pulmonary TB are sputum smear negative and thus microscopy is insufficient to provide accurate diagnosis. ${ }^{2}$ Moreover, about $20 \%$ of all patients with TB are sputumnegative and culture-negative and must be diagnosed using clinical examination and response to anti-TB treatment. Consequently, TB diagnosis is highly problematic in small children and in patients with suspected extra-pulmonary TB, HIV infection or other immunosuppressive diseases. Furthermore, none of the existing commercial methods clearly separates active TB disease from latent infection, which makes it difficult to select the appropriate chemotherapy.

Antibodies in Lymphocyte Supernatant (ALS) is a noncommercial method that has previously been developed and applied in diagnosis of active pulmonary $\mathrm{TB}^{3-7}$ This method detects antigen-specific antibodies secreted by peripheral blood mononuclear cells (PBMCs) and has also been used to assess mucosal immune responses to oral cholera ${ }^{8}$ and typhoid ${ }^{9}$ vaccines, and in patients with enterotoxigenic Escherichia coli (ETEC) diarrhoea. ${ }^{10}$ In contrast to conventional serology, ${ }^{11} 12$ which involves assessment of stable serum antibodies, ${ }^{7}$ the ALS test is based on the spontaneous release of BCG-specific IgG antibodies from peripheral plasmablasts temporarily present in the blood. ${ }^{3-5}$ Our hypothesis is that pathogen-specific antibody secreting cells (ASCs) are only present in blood during active or subclinical disease ${ }^{13}{ }^{14}$ and not during latent infection or under healthy conditions. ${ }^{15}$ To explore whether BCG-specific IgG antibodies secreted by peripheral plasmablasts could be used as a host-specific biomarker to detect different clinical forms of active TB disease among patients who are HIV negative or HIV positive, we assessed the activity of ASCs in blood samples from Ethiopian individuals with sputum smear negative TB.

\section{METHODS}

\section{Study subjects}

Participants were recruited at the Chest Unit, Black Lion University Hospital, Addis Ababa, Ethiopia after providing signed informed consent. The study was approved by the national ethical committees in Ethiopia and Sweden. Inclusion criteria were individuals who were HIV negative or HIV positive and sputum smear negative, over 18 years of age with clinical symptoms of suspected TB. Exclusion criteria were patients with a history of previous TB or more than 1 week of antimicrobial chemotherapy, those who used antiretroviral drugs or did not consent to HIV screening. Asymptomatic individuals with no clinical disease were recruited as controls.

Clinical diagnosis was based on typical TB symptoms (persistent cough and general illness including fever, weight and appetite loss, and sweating for 1-5 months, pleural effusions or chronic non-tender cervical lymphadenopathy $>6$ weeks), chest $\mathrm{x}$-ray and positive response to anti-TB treatment (clinical improvement and radiographical resolution of pulmonary TB lesions). Active TB disease was confirmed by a clinical diagnosis of $\mathrm{TB}$ and/or positive Mtb culture or cyto-histopathology of clinical specimens. Blood samples obtained from the subjects at the time of diagnosis were used for the QFTG and ALS assays, peripheral CD3/CD4 T-cell counts (FACSCount; BD Biosciences, Franklin Lakes, New Jersey, USA) and to determine HIV status. A detailed description of study subjects, clinical and immunological diagnoses, standard care and HIV testing can be found in the online supplementary material.

\section{Tuberculin skin test}

The TST measures the presence of delayed-type hypersensitivity in the skin upon intra-dermal injection of $0.1 \mathrm{ml}$ Tuberculin
Purified Protein Derivative (PPD) (5TU; SSI, Copenhagen, Denmark) in the volar aspect of the forearm. The TST reaction, measured by trained research nurses at $48-72 \mathrm{~h}$ after tuberculin injection, was considered positive when the transverse induration was $\geq 10 \mathrm{~mm}(\geq 5 \mathrm{~mm}$ for subjects who were HIV positive).

\section{QuantiFERON-TB Gold in-Tube}

The QFTG assay measures IFN $\gamma$ production by $\mathrm{T}$ cells after in vitro stimulation of whole blood with the Mtb-specific antigens, CFP-10, ESAT-6, TB7.7, according to the manufacturer's (Cellestis; SSI) instructions. QFTG values above a cut-off of $0.35 \mathrm{IU} / \mathrm{ml}$ were considered positive.

\section{Antibodies in lymphocyte supernatant}

The ALS assay measures spontaneous release of BCG-specific IgG antibodies from in vivo-derived plasmablasts using in vitro cultures of unstimulated PBMCs and an ELISA ${ }^{3-6}$ as described in the online supplementary material. Briefly, PBMCs were isolated from 3 to $5 \mathrm{ml}$ of blood using cell preparation tubes (CPT; $\mathrm{BD}$ Biosciences) and cultured $\left(2.5 \times 10^{6}\right.$ cells $/ \mathrm{ml}$ in 48 -well plates) in RPMI medium (GIBCO, Invitrogen; Carlsbad, California, USA) for $72 \mathrm{~h}$. Release of IgG antibodies in the culture supernatant was measured using a BCG-specific ELISA. The ASC response is expressed as relative BCG-specific IgG titres and OD (optical density) values above a cut-off of 0.425 were considered positive.

\section{Flow cytomety}

Randomly selected PBMC samples $\left(5-10 \times 10^{6}\right.$ cells) from patients with active TB $(n=19)$, those with latent TB $(n=7)$ and non-TB controls $(n=6)$ were frozen in $1 \mathrm{ml}$ of RPMI with $20 \%$ fetal calf serum (FCS) and 10\% dimethyl sulfoxide (Sigma-Aldrich, St Louis, Missouri, USA) and stored at $-150^{\circ} \mathrm{C}$ until flow cytometric analysis. After thawing, rested PBMCs were washed with phosphate buffered saline $0.1 \%$ FCS and stained for $15 \mathrm{~min}$ at $4^{\circ} \mathrm{C}$ with the following antibodies: CD3 Pacific Blue, CD20 APC Cy7, CD27 PE, CD38 APC, CD19 PE and IgG PE Cy5 (BD Biosciences). After fixation with 1\% paraformaldehyde (Sigma-Aldrich) for $30 \mathrm{~min}$ at $4^{\circ} \mathrm{C}$, PBMCs were analysed using a Gallios flow cytometer and the Kaluza software (Beckman Coulter, Brea, California, USA; online supplementary material, figure 1).

\section{Statistical analysis}

Non-parametric analyses were used to calculate $\mathrm{p}$ values and included a Kruskal-Wallis test and Dunn's post test or a MannWhitney test. Spearman's correlation test was used for the correlation analysis. Receiver operating characteristic curves were used to determine the relation between sensitivity and specificity at various cut-off levels of BCG-specific IgG titres (online supplementary material, figure 2). Selection of the best cut-off point (OD 0.425) was based on the level of maximum accuracy. Cohen's $\kappa$ coefficient and McNemar's $\chi^{2}$ test were used to determine the diagnostic agreement between the ALS assay and clinical diagnosis. Statistical analyses were performed using GraphPad Prism-4 and SPSS V.12.

\section{RESULTS}

\section{Characterisation of study subjects}

The demographics of the study subjects are outlined in table 1 . Among 96 suspected TB cases, clinical and pathologicalanatomical diagnoses, and $\mathrm{Mtb}$ culture were used to confirm active TB disease in 84 patients (table 1). Patients with active TB 

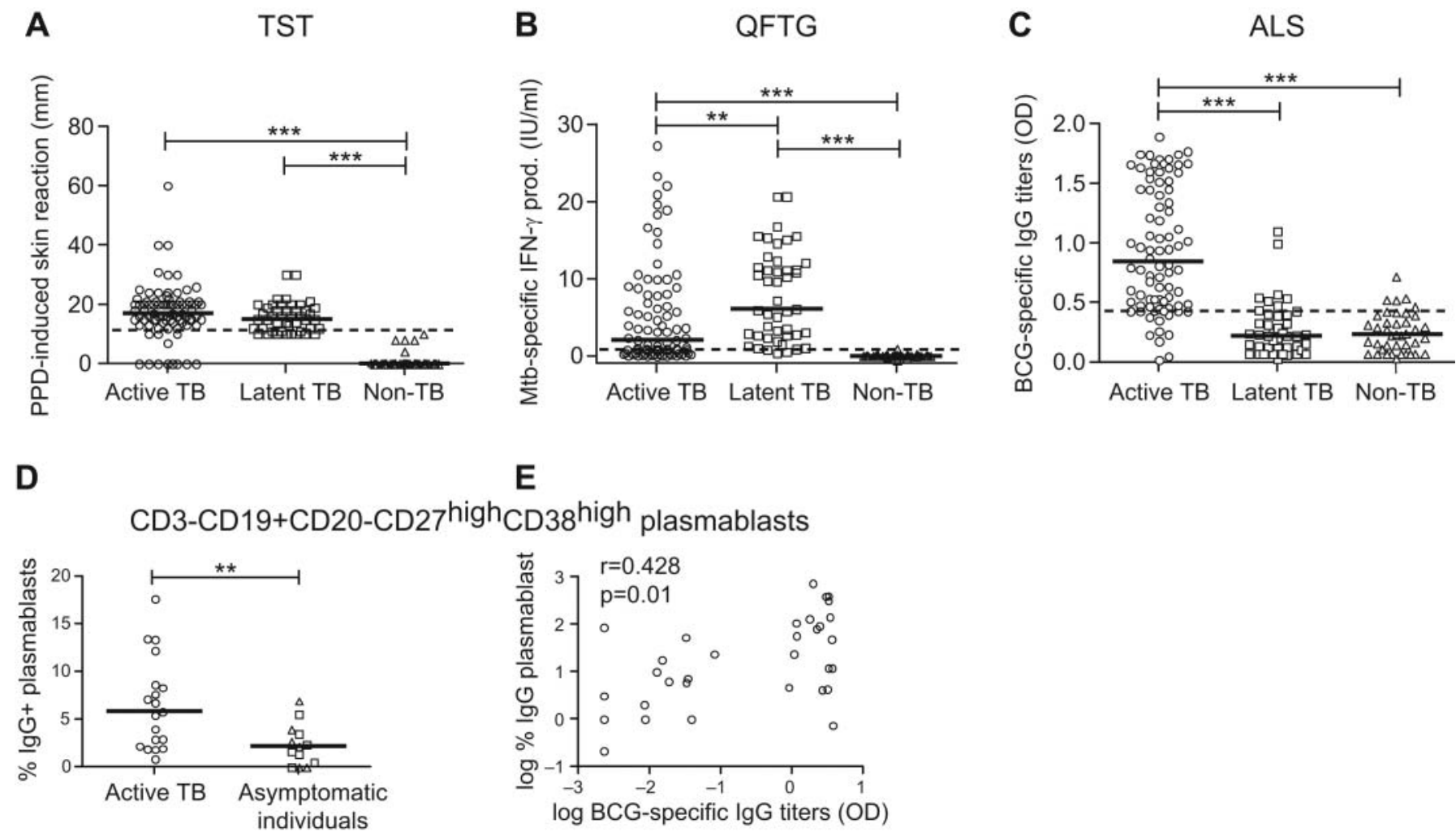

Figure 1 Assessment of BCG-specific lgG antibodies secreted by peripheral plasmablasts in patients with active tuberculosis (TB) (circles) compared with those with latent TB (squares) and non-TB controls (triangles). The graphs show results from (A) the tuberculin skin test (TST) (skin induration), (B) QuantiFERON-TB Gold in-Tube (QFTG) (interferon (IFN)- $\gamma$ production) and (C) the Antibodies in Lymphocyte Supernatant (ALS) (IgG titres) among the different groups of patients. The dashed lines indicate the positive cut-off level determined for each diagnostic test: PPD $\geq 10$ $(\mathrm{mm})$, IFN $\gamma \geq 0.35$ (IU/ml) and IgG titres $\geq 0.425$ (OD). (D) The proportion of IgG+ CD3-CD19+CD20-CD27 $7^{\text {high }} \mathrm{CD} 38^{\text {high }}$ plasmablasts among PBMCs was determined by flow cytometric analysis of samples obtained from patients with active TB and asymptomatic individuals. (E) Correlation analysis between IgG+ plasmablasts (PBMC samples) and the corresponding BCG-specific lgG titres (PBMC culture supernatants) among the study subjects. Graphs are presented as scatter dot plots and the solid bars indicate the median values for each group. The statistical significance of differences in diagnostic performance between the different patients groups was determined using the Kruskal-Wallis test (A-C) or a Mann-Whitney test (D). ${ }^{*} p<0.05,{ }^{* *} p<0.01$ and ${ }^{* * *} p<0.001$. Spearman's correlation test was used to determine the correlation coefficient $r_{s}(E)$. A value of $r=1$, indicates a perfect positive correlation whereas $r=-1$ indicates a perfect negative or inverse correlation.

A

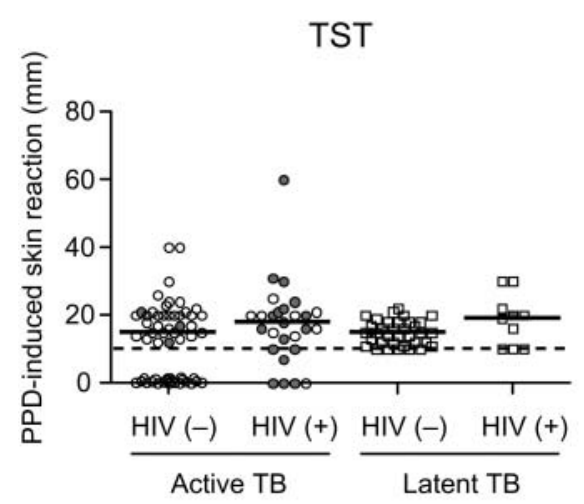

B

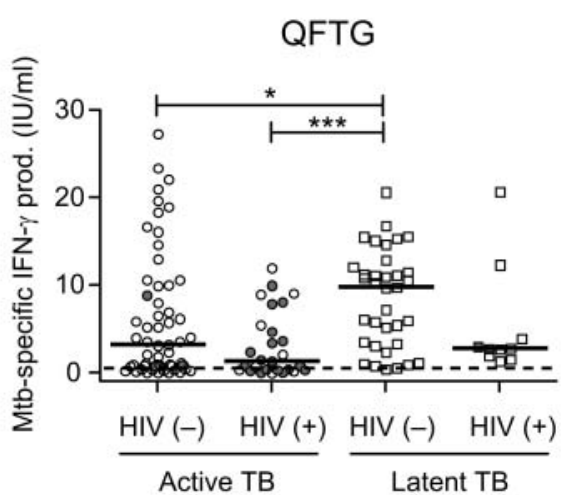

C

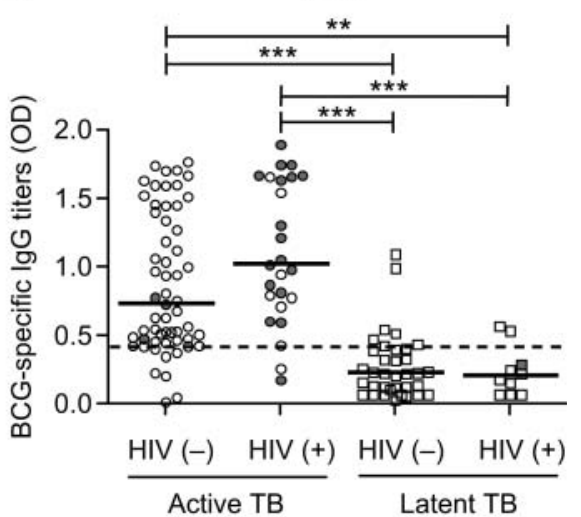

Figure 2 Assessment of BCG-specific IgG antibodies secreted by peripheral plasmablasts in patients who are HIV negative compared with those who are HIV positive with either active tuberculosis (TB) (circles) or latent TB (squares). Red symbols represents patients with a CD4 T-cell count $<200$ cells/ml. The graphs show results from (A) the tuberculin skin test (TST) (skin induration), (B) QuantiFERON-TB Gold in-Tube (QFTG) (interferon (IFN) $-\gamma$ production) and (C) the Antibodies in Lymphocyte Supernatant (ALS) (IgG titres) among the different groups of patients. The dashed lines indicate the positive cut-off level determined for each diagnostic test: PPD $\geq 10$ (mm), IFN $\gamma \geq 0.35$ (IU/ml) and IgG titres $\geq 0.425$ (OD). All graphs are presented as scatter dot plots and the solid bars indicate the median values for each group. The statistical significance of differences in diagnostic performance between the different patients groups was determined using the Kruskal-Wallis test $(A$ and $B) .{ }^{*} p<0.05,{ }^{* *} p<0.01$ and ${ }^{* * *} p<0.001$. 
Table 1 Clinical demographics of included study subjects

\begin{tabular}{|c|c|c|c|c|c|c|c|}
\hline \multirow[b]{2}{*}{ Clinical features, n (\%) } & \multicolumn{5}{|c|}{ Symptomatic TB suspects } & \multicolumn{2}{|c|}{ Asymptomatic individuals } \\
\hline & $\begin{array}{l}\text { Total active TB } \\
(n=84)\end{array}$ & $\begin{array}{l}\text { Pulmonary TB } \\
(n=35)\end{array}$ & $\begin{array}{l}\text { Pleural TB } \\
(n=23)\end{array}$ & $\begin{array}{l}\text { Lymph node TB } \\
(n=26)\end{array}$ & $\begin{array}{l}\text { Other diseases* } \\
(n=12)\end{array}$ & $\begin{array}{l}\text { Latent TB } \\
(\mathrm{n}=45)\end{array}$ & $\begin{array}{l}\text { Non-TB controls } \\
(n=40)\end{array}$ \\
\hline $\begin{array}{l}\text { Median age in years } \\
\text { (range) }\end{array}$ & $27(18-72)$ & $27(18-54)$ & $28(18-72)$ & $26(18-57)$ & $39(20-55)$ & $29(18-60)$ & $28(18-68)$ \\
\hline Men/women & $45 / 39$ & $20 / 15$ & $11 / 12$ & $14 / 12$ & $5 / 7$ & $29 / 16$ & $13 / 27$ \\
\hline Abnormal chest x-ray $\dagger$ & $58(69 \%)$ & $35(100 \%)$ & $23(100 \%)$ & $0(0 \%)$ & $7(58 \%)$ & $0(0 \%)$ & $0(0 \%)$ \\
\hline Positive Mtb culture $\ddagger$ & $24(29 \%)$ & $24(69 \%)$ & ND & ND & $0(0 \%)$ & ND & ND \\
\hline $\begin{array}{l}\text { Positive Mtb } \\
\text { cyto-histopathology }\end{array}$ & $49(58 \%)$ & ND & $23(100 \%)$ & $26(100 \%)$ & $0(0 \%)$ & ND & ND \\
\hline Positive TST $\geq 10 \mathrm{~mm}$ & $75(89 \%)$ & $30(86 \%)$ & $20(87 \%)$ & $25(96 \%)$ & $3(25 \%)$ & $45(100 \%)$ & $0(0 \%)$ \\
\hline $\begin{array}{l}\text { Positive QFTG (cut-off } \\
0.35) \S\end{array}$ & $66(79 \%)$ & $25(71 \%)$ & $17(74 \%)$ & $24(92 \%)$ & $3(25 \%)$ & $45(100 \%)$ & $0(0 \%)$ \\
\hline HIV infection & $27(32 \%)$ & $12(34 \%)$ & $7(30 \%)$ & $8(31 \%)$ & $4(33 \%)$ & $10(22 \%)$ & $22(55 \%)$ \\
\hline Parasite infections & $9(11 \%)$ & $4(11 \%)$ & $1(4.3 \%)$ & $4(15 \%)$ & $0(0 \%)$ & ND & ND \\
\hline BCG vaccination & $17(20 \%)$ & $10(29 \%)$ & $5(22 \%)$ & $2(7.7 \%)$ & $3(25 \%)$ & $12(27 \%)$ & $10(25 \%)$ \\
\hline Disease outcome (cured)** & $62(74 \%)$ & $24(69 \%)$ & $20(87 \%)$ & $18(69 \%)$ & ND & ND & ND \\
\hline
\end{tabular}

(median age 27 years, men/women: 45/39) were further divided into three groups based on different clinical forms of the disease: pulmonary TB $(n=35)$ or extra-pulmonary pleural TB $(\mathrm{n}=23)$ and lymph node TB without pulmonary involvement $(\mathrm{n}=26)$ (table 1). According to histopathology and response to conventional antibiotic treatment, diseases other than TB were diagnosed in 12 symptomatic patients (table 1 ). Three of these patients had latent TB as suggested by a positive TST and QFTG test (table 1 ). Among 85 asymptomatic individuals (median age: 28.5 years, men/women: $42 / 43$ ), 45 cases with positive TST and QFTG tests were grouped as latent TB, while 40 individuals who were negative for both these tests were grouped as non-TB controls (table 1). On average, one-third of the study subjects were infected with HIV (table 1).

\section{Elevated levels of circulating BCG-specific IgG-secreting plasmablasts were detected in patients with active TB disease}

The majority of patients with active TB had a positive TST $(89 \%)$ and/or a positive QFTG (79\%) test result and consequently these tests could not discriminate active from latent TB (table 1 and figure 1A,B). In contrast, the ALS test revealed that BCG-specific IgG secretion from circulating plasmablasts could be detected in most patients with active TB (91\%) but only in a few latent TB cases (16\%) (figure 1C). Importantly, median IgG titres were significantly higher in active TB compared with other diseases $(\mathrm{p}<0.01$; data not shown) or latent TB and non-TB controls $(p<0.001)$, which suggests that BCG-specific ASC are only present in the circulation of patients with active TB disease (figure 1C). In line with this finding, multicolour flow cytometric analyses of PBMC samples demonstrated that the proportion of circulating CD3-CD19+CD20-CD2 $7^{\text {high }} \mathrm{CD} 38^{\text {high }}$ plasmablasts expressing cell-surface $\operatorname{IgG}^{16}$ was significantly $(\mathrm{p}<0.01)$ higher among PBMCs from patients with active TB $(n=19)$ compared with asymptomatic individuals $(n=13)$, which included those with latent TB and non-TB controls (figure 1D). Importantly, there was a significant correlation $(r=0.428$, $\mathrm{p}=0.01$ ) between IgG+ plasmablasts in PBMC samples and BCG-specific IgG antibodies secreted by the PBMCs as determined by the ALS assay (figure 1E).

\section{High BCG-specific IgG titres were associated with low Mtb-specific IFN $\gamma$ levels in patients with HIV infection and active TB disease}

Among subjects who were HIV negative or HIV positive with either active or latent TB, CD4 T-cell counts $<200$ cells $/ \mathrm{ml}$ (figure 2, red symbols) were primarily found in those with HIV and active TB (81\%). As expected, Mtb-specific IFN $\gamma$ production in vitro was low in blood samples from patients with $\mathrm{TB} /$ HIV coinfection, while IFN $\gamma$ levels were significantly $(p<0.001)$ higher in samples from subjects who were HIV negative and had latent TB (figure 2B). In contrast, BCG-specific IgG titres were significantly $(p<0.001)$ higher in the TB/HIV coinfected group compared with individuals who were HIV negative or HIV positive with latent TB (figure 2C). Consequently, the sensitivity $(80-100 \%)$ and specificity $(78-94 \%)$ of the ALS test to detect active TB was generally high among patients who were HIV negative or HIV positive, particularly among those with HIV and pulmonary or lymph node TB (table 2). The positive predictive values of the ALS assay were high in most TB groups, but relatively lower among patients who were HIV positive with pleural TB (table 2). The specificity and positive predictive values were also relatively lower comparing active TB with latent TB cases than comparing active TB with non-TB controls (table 2). The negative predictive values were consistently high in all groups (table 2).

Furthermore, we found that the overall agreement between the ALS assay and clinical TB diagnosis (confirmed and clinical 
Table 2 Diagnostic performance of the ALS test*

\begin{tabular}{|c|c|c|c|c|c|c|c|c|}
\hline \multirow[b]{2}{*}{ Groups } & \multicolumn{4}{|c|}{ Latent TB cases $(n=45)$} & \multicolumn{4}{|c|}{ Non-TB controls $(n=40)$} \\
\hline & Sensitivity & Specificity & PPV & NPV & Sensitivity & Specificity & PPV & NPV \\
\hline \multicolumn{9}{|l|}{ All TB cases } \\
\hline HIV negative & 86 & 80 & 89 & 86 & 86 & 94 & 98 & 74 \\
\hline HIV positive & 90 & 80 & 94 & 73 & 91 & 94 & 96 & 90 \\
\hline \multicolumn{9}{|l|}{ Pulmonary TB } \\
\hline HIV negative & 80 & 80 & 79 & 88 & 80 & 94 & 95 & 81 \\
\hline HIV positive & 86 & 80 & 85 & 89 & 90 & 94 & 92 & 94 \\
\hline \multicolumn{9}{|l|}{ Pleural TB } \\
\hline HIV negative & 80 & 80 & 75 & 97 & 87 & 94 & 94 & 94 \\
\hline HIV positive & 86 & 80 & 67 & 89 & 80 & 91 & 80 & 94 \\
\hline \multicolumn{9}{|l|}{ Lymph node TB } \\
\hline HIV negative & 86 & 80 & 77 & 97 & 84 & 94 & 94 & 94 \\
\hline HIV positive & 100 & 78 & 80 & 100 & 100 & 91 & 88 & 94 \\
\hline
\end{tabular}

TB cases) was very good $(\kappa>0.742 ; \mathrm{p}>0.625)$ among all active TB patients, with or without HIV infection (table 3 ).

\section{Elevated levels of BCG-specific IgG-secreting plasmablasts correlated with reduced T-cell counts and progression of disease}

Total T-cell counts determined in peripheral blood of the study subjects revealed that the levels of CD3 T cells (figure $3 \mathrm{~A}$ ), and CD4 and CD8 T cells (data not shown), were clearly lower in patients with active TB compared with the other groups, particularly when compared with latent TB cases $(p<0.001)$. We also observed a significant inverse correlation $(\mathrm{r}=-0.311$, $\mathrm{p}=0.004$ ) between CD3 T-cell counts and BCG-specific IgG titres among patients with active TB (figure 3B) but not among asymptomatic individuals (data not shown). CD4 T-cell numbers were also significantly $(\mathrm{p}<0.05)$ lower in active TB patients who were HIV negative or HIV positive compared with individuals with latent TB (figure 3C). Accordingly, there was a significant correlation $(r=0.303, p=0.042)$ between CD4 T cells and Mtb-specific IFN $\gamma$ production among individuals with latent TB (figure 3D) but not among patients with active TB disease (data not shown).

Table 3 Concordance and agreement between the ALS test and clinical diagnosis among patients with active TB

\begin{tabular}{llll}
\hline Groups & Concordance & $\begin{array}{l}\text { Agreement, } \\
\text { Cohen's к (SE) }\end{array}$ & $\begin{array}{c}\text { p Value, } \\
\text { McNemar }\end{array}$ \\
\hline $\begin{array}{l}\text { All TB cases } \\
\text { HIV negative }\end{array}$ & $68 / 75(90.7 \%)$ & $0.766(0.083)$ & 0.725 \\
HIV positive & $41 / 47(87.2 \%)$ & $0.742(0.098)$ & 0.687 \\
$\begin{array}{l}\text { Pulmonary TB } \\
\text { HIV negative }\end{array}$ & $36 / 41(87.8 \%)$ & $0.804(0.093)$ & 0.625 \\
HIV positive & $29 / 34(85.3 \%)$ & $0.861(0.095)$ & 1.00 \\
$\begin{array}{l}\text { Pleural TB } \\
\text { HIV negative }\end{array}$ & $32 / 34(94.1 \%)$ & $0.882(0.081)$ & 1.00 \\
HIV positive & $22 / 27(81.5 \%)$ & $0.744(0.171)$ & 1.00 \\
Lymph node TB & & & 1.00 \\
HIV negative & $34 / 36(94.4 \%)$ & $0.889(0.076)$ & 1.00 \\
HIV positive & $26 / 30(86.7 \%)$ & $0.913(0.085)$ & \\
\hline ALS, Antibodies in Lymphocyte Supernatant; TB, tuberculosis. &
\end{tabular}

Comparing different clinical forms of active TB, peripheral CD3 T-cell counts were significantly $(\mathrm{p}<0.05)$ higher in patients with local lymph node TB compared with pleural TB (figure 3E). Likewise, Mtb-specific IFN $\gamma$ production in vitro was significantly higher in patients with TB lymphadenitis compared with those with pulmonary or pleural $\mathrm{TB}(\mathrm{p}<0.001$ and $\mathrm{p}<0.05$, respectively) (figure 3F). There was no difference in BCG-specific IgG titres between these groups (data not shown). Patients with TB lymphadenitis also showed a significant correlation $(\mathrm{r}=0.520, \mathrm{p}=0.007)$ between CD4 T-cell counts and Mtb-specific IFN $\gamma$ production (figure $3 \mathrm{G}$ ), but a significant negative correlation $(r=-0.429, p=0.03)$ between CD4 T cells and BCG-specific IgG titres (figure $3 \mathrm{H}$ ). Of note, HIV-infected patients with TB lymphadenitis (red symbols) who had low CD4 T-cell counts expressed relatively lower levels of IFN $\gamma$ but higher IgG levels compared with HIV negative patients with TB lymphadenitis (open symbols) who had higher CD4 T-cell counts (figure $3 \mathrm{G}-\mathrm{H}$ ).

\section{DISCUSSION}

Improved diagnosis and treatment of clinical TB in high-risk groups would have a great impact on preventing the global spread of disease. Here, we explored TB immunodiagnosis using the ALS test, based on assessment of BCG-specific IgG-secreting plasmablasts in peripheral blood samples from patients with sputum-negative TB and asymptomatic individuals. This study provides evidence that circulating $\operatorname{IgG}+$ plasmablasts and spontaneous secretion of BCG-specific IgG antibodies were significantly higher in patients with active TB compared with individuals with latent TB and non-TB controls. BCG-specific IgG titres were particularly high among patients with TB/HIV coinfection and CD4 T-cell counts $<200$ cells/ml who produced low levels of Mtb-specific IFN $\gamma$ in vitro. Hence, elevated ASC responses were generally associated with impaired peripheral $\mathrm{T}$-cell responses, including reduced $\mathrm{T}$-cell numbers and low Mtb-specific IFN $\gamma$ production. These results suggest that detection of BCG-specific IgG-secreting plasmablasts could be successfully used as a diagnostic biomarker to detect different clinical forms of sputum-negative TB and distinguish active TB from latent TB infection in patients who are HIV negative, and particularly, in those with TB/HIV coinfection.

In the steady state, the proportion of migratory and IgG-secreting plasmablasts among PBMCs is very low, but 

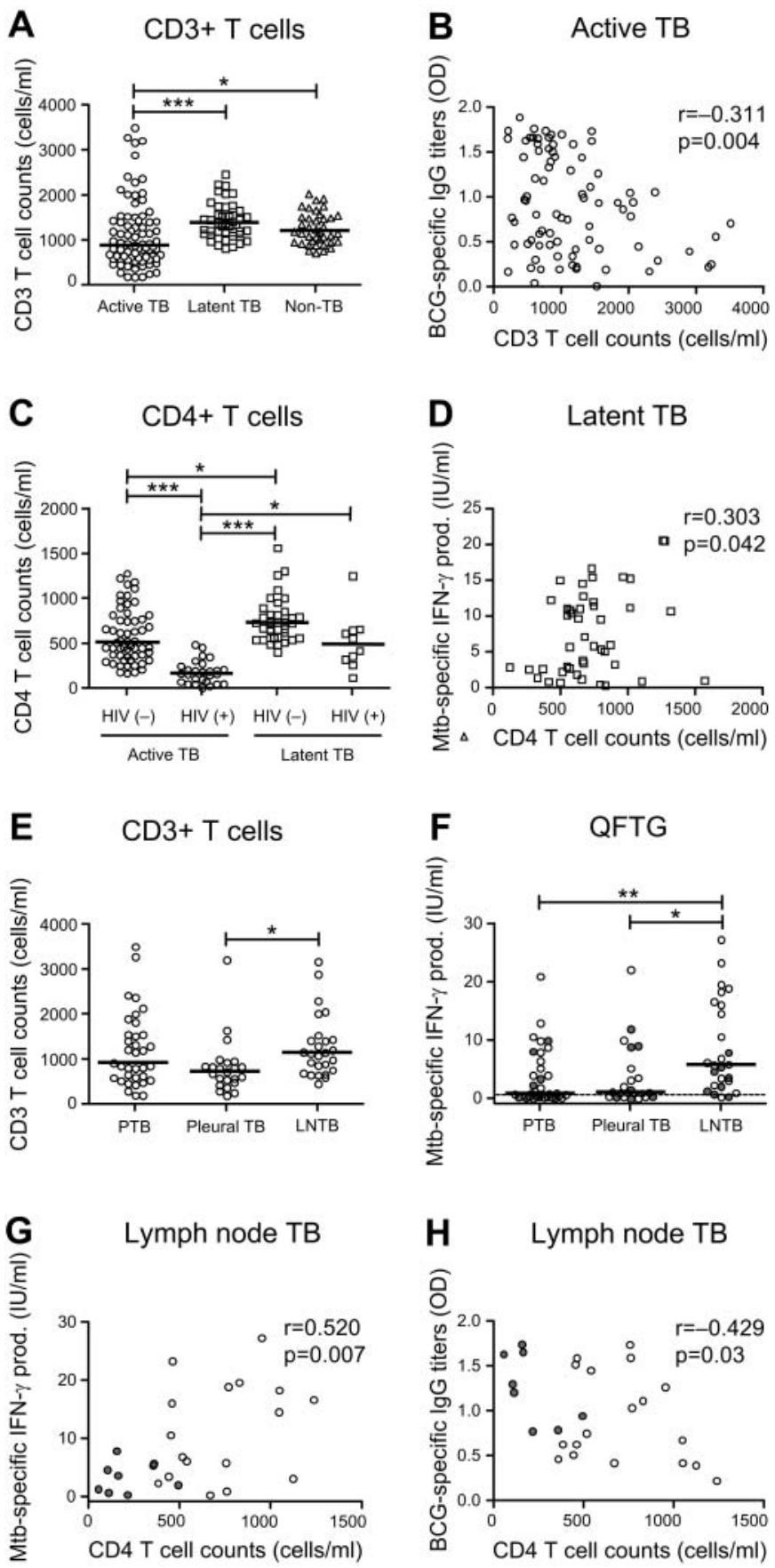

Figure 3 Assessment and comparison of peripheral blood T-cell counts, BCG-specific lgG titres and Mycobacterium tuberculosis (Mtb)-specific interferon (IFN)- $\gamma$ production in patients with different clinical forms of tuberculosis (TB). Red symbols represent patients with HIV infection. (A) Total peripheral CD3 T-cell counts were determined in cases with active TB (circles), latent TB (squares), and non-TB controls (triangles). (B) Correlation analysis of peripheral blood CD3 T-cell counts and BCG-specific lgG titres among patients with active TB (circles). (C) Peripheral CD4 T-cell counts in patients who were HIV negative or HIV positive with active TB (circles) or latent TB (squares). (D) Correlation analysis of peripheral blood CD4 T-cell counts and Mtb-specific IFN $\gamma$ production in vitro among patients with latent TB (squares). (E) Total peripheral CD3 T-cell counts and (F) Mtb-specific IFN $\gamma$ production in vitro in patients with pulmonary TB (PTB), pleural TB or lymph node TB (LNTB) are shown. Correlation analysis of peripheral blood CD4 T-cell counts and (G) Mtb-specific IFN $\gamma$ production in vitro or $(\mathrm{H}) \mathrm{BCG}$-specific lgG titres among patients who were HIV negative (open symbols) or HIV positive (red symbols) with TB significantly elevated upon the continuous antigen exposure resulting after systemic vaccination. ${ }^{13}{ }^{15}$ Consequently, ASCs should be temporarily present in the peripheral circulation only in patients with active TB disease. ${ }^{14}$ By contrast, effector memory $\mathrm{T}$ cells persist in the blood ${ }^{17}$ of individuals with active or latent TB, and consequently, neither the TST nor the IFN $\gamma$ release assay can discriminate active from latent TB infection. ${ }^{18}$ It is possible that the few latent $\mathrm{TB}$ cases with elevated BCG-specific IgG titres may indicate subclinical TB infection associated with an increased risk of developing active TB. ${ }^{4}$ It was recently demonstrated that the speed, sensitivity and specificity of the ALS assay can be enhanced by using higher numbers of PBMCs in a concentrated 96-well format (micro-ALS method). ${ }^{5}$ This set-up may increase the possibility of detecting borderline cases, with BCG-specific IgG titres close to the cut-off level.

Previous studies have demonstrated that patients with respiratory diseases other than TB possessed significantly lower BCG-specific IgG titres compared with patients with TB. ${ }^{3} 56$ Future studies should also systematically evaluate the ALS test in patients with pulmonary TB compared with patients with respiratory diseases such as bacterial pneumonias, pulmonary malignancies, aspergillosis, bronchiectasis or pleural empyemas, which represents clinical conditions commonly encountered in developing countries that may pose a serious problem to the differential diagnosis. Recently, it was also shown that the BCG vaccine is a superior antigen for detection of ASC in PBMC samples from patients with TB in comparison with a panel of Mtb-specific antigens, including LAM, ESAT-6, CFP-10, TB15.3 and TB51A. ${ }^{5}$ However, further development of the ALS assay using cocktails of Mtb-specific peptide pools may significantly improve the specificity of this test.

To date, most commercial and novel immunological and microbiological assays fail to significantly improve diagnosis of active TB in high-risk groups. Promising results have been obtained using enzyme-linked immunospot ${ }^{19}$ or microscopic-observation drug-susceptibility ${ }^{20}$ assays. However, these methods require invasive patient sampling techniques ${ }^{19}$ or advanced microscopy and time-consuming work with hazardous mycobacterial cultures, ${ }^{20}$ and fail to demonstrate efficient diagnostic results in people with $\mathrm{TB} / \mathrm{HIV}$ coinfection $^{19}$ and those who are sputum smear negative, ${ }^{20} 21$ respectively. In addition, conventional serological assays have not been successful in the diagnosis of sputum smear-negative $\mathrm{TB}$ or in differentiating active from latent infection. ${ }^{7}{ }^{12}$ Stable serum antibodies are continuously present in the circulation to provide long-term host protection and are primarily produced by non-migratory and long-lived plasma B cells that reside in the bone marrow. ${ }^{22} 23$ High levels of total and Mtb-specific serum antibodies have previously been shown in patients with severe forms of $\mathrm{TB}$ disease, ${ }^{2425}$ but the majority of studies reveal highly variable results and suboptimal sensitivity of serology in sputum-negative and sputum-positive patient groups and, in particular, among those who are HIV positive. ${ }^{11} 26-28$

lymphadenitis. Graphs are presented as scatter dot plots and the solid bars indicate the median values for each group whereas the dashed lines indicate the positive cut-off level determined for IFN $\gamma \geq 0.35$ (IU/ $\mathrm{ml}$ ) and lgG titres $\geq 0.425$ (OD). T-cell counts are presented as cells $/ \mathrm{ml}$. Statistical analyses included the Kruskal-Wallis and Spearman's correlation tests. A value of $r=1$ for the correlation coefficient $r_{s}$ indicates a perfect positive correlation whereas $r=-1$ indicates a perfect negative or inverse correlation. ${ }^{*} p<0.05,{ }^{*} p<0.01$ and $* * * p<0.001$. 
Here we provide evidence that the ALS test could be useful to detect active TB among patients who are sputum-negative and those who are immunosuppressed with low CD4 T-cell counts and low QFTG responses. The TST and the QFTG tests depend on the absolute numbers of circulating CD4 T cells, ${ }^{29}$ and consequently, demonstrate a significantly reduced sensitivity for the diagnosis of active TB in immunodeficient individuals who often present anergic antigen-specific T-cell responses. ${ }^{18}$ 30-32 Instead, increased CD4 T-cell counts correlated with elevated Mtb-specific IFN $\gamma$ responses in latent TB (ie, control of TB disease) and in HIV negative patients with local lymph node TB (ie, mild TB disease), while TB/HIV coinfection (ie, advanced TB disease) was associated with reduced CD4 T-cell counts and enhanced secretion of BCG-specific IgG antibodies from peripheral plasmablasts. Importantly, it has been shown that BCG-specific IgG titres gradually decrease after 2 and 6 months of successful anti-TB therapy, but remain high in patients infected with drug-resistant TB, also indicating that high levels of ASCs are maintained during progressive TB. ${ }^{4} 67$

Interestingly, HIV infection could give rise to different types of B-cell abnormalities, including selective loss of antigenspecific memory $\mathrm{B}$ cells ${ }^{33}$ but also hypergammaglobulinemia and the production of polyclonal antibodies by activated naïve B cells. ${ }^{34}$ Hypergammaglobulinemia has also been observed in patients who are HIV negative and in HIV positive patients with TB or Mycobacterium leprae infection. ${ }^{24}$ It is likely that continuous exposure of viral and bacterial antigens in chronic infections with mycobacteria and/or HIV may enhance B-cell activation and antibody-mediated immunity, particularly in patients with impaired T-cell responses. Similarly, in patients with worm infection, reduced CD4 T-cell numbers in blood correlated with enhanced levels of worm-specific $\operatorname{IgG}$ antibodies and severity of disease, especially when the release of antigens from pathological lesions was augmented. ${ }^{35}$

Together, our findings suggest that assessment of BCG-specific IgG antibodies secreted by plasmablasts in the peripheral circulation could be exploited as an efficient biomarker to improve diagnosis of sputum smear-negative active TB among patients who are HIV negative or HIV positive. Development and clinical implementation of a rapid and simple point-of-care immunodiagnostic test such as the ALS assay could be very useful for TB control programmes in developing and industrialised countries.

\footnotetext{
Author affiliations

${ }^{1}$ Department of Medicine, Center for Infectious Medicine (CIM), Karolinska Institutet, Karolinska University Hospital Huddinge, Stockholm, Sweden

${ }^{2}$ Department of Pathology, Faculty of Medicine, Black Lion University Hospital and Addis Ababa University, Addis Ababa, Ethiopia

${ }^{3}$ Department of Internal Medicine, Faculty of Medicine, Black Lion University Hospital and Addis Ababa University, Addis Ababa, Ethiopia

${ }^{4}$ Armauer Hansen Research Institute (AHRI), Addis Ababa, Ethiopia

${ }^{5}$ Laboratory Science Division, International Centre for Diarrhoeal Disease Research, Bangladesh (ICDDR,B), Dhaka, Bangladesh

${ }^{6}$ Department of Microbiology, Tumor and Cell Biology (MTC), Karolinska Institutet, Karolinska University Hospital Huddinge, Stockholm, Sweden

${ }^{7}$ Department of Surgery, Faculty of Medicine, Black Lion University Hospital and Addis Ababa University, Addis Ababa, Ethiopia

${ }^{8}$ Department of Radiology, Faculty of Medicine, Black Lion University Hospital and Addis Ababa University, Addis Ababa, Ethiopia

${ }^{9}$ Division of Infectious Diseases, Department of Medicine, Karolinska University Hospital Huddinge, Stockholm, Sweden
}

Acknowledgements We thank the research nurses, laboratory staff and technicians at the Black Lion University Hospital and Armauer Hansen Research Institute, Addis Ababa, Ethiopia, for excellent collaboration and support during this study. We also thank Dr Mesfin Kassaye at Karolinska Institutet for assistance with the statistical calculations and Dr Steven E Applequist at Karolinska Institutet for critical reading of the manuscript.
Contributors All authors contributed substantially to this study. Conception and design: SA, GAd, RR, JA, SB; patient recruitment, clinical diagnosis and clinical care including acquisition of clinical data, analysis and interpretation of clinical data: $\mathrm{GAd}, \mathrm{AB}, \mathrm{BL}, \mathrm{GAs}$; laboratory work including acquisition of data, analysis and interpretation of data: $S A, M Z, R R$, IM, MH, RSR, MM, AA, MS, JA, SB; preparation and review of manuscript: $S A, R R, M S, J A, S B$.

Funding This work was supported by funding from the Swedish research council (VR), the Heart and Lung Foundation (HLF), the Swedish Society for Medical Research (SSMF), the Swedish International Development Cooperation Agency (Sida), the Swedish Civil Contingencies Agency (MSB) and the Swedish Foundation for Strategic Research (SSF).

\section{Competing interests None.}

Ethics approval Ethics approval was provided by the Regional Ethical Review Board in Stockholm, Sweden (EPN) and the National Health Research Ethics Committee (NERC) in Ethiopia.

Provenance and peer review Not commissioned; externally peer reviewed.

Open Access This is an open-access article distributed under the terms of the Creative Commons Attribution Non-commercial License, which permits use, distribution, and reproduction in any medium, provided the original work is properly cited, the use is non commercial and is otherwise in compliance with the license. See: http://creativecommons.org/licenses/by-nc/2.0/ and http://creativecommons.org/ licenses/by-nc/2.0/legalcode.

\section{REFERENCES}

1 Wallis RS, Pai M, Menzies D, et al. Biomarkers and diagnostics for tuberculosis: progress, needs, and translation into practice. Lancet 2010;375:1920-37.

2 Siddiqi K, Lambert ML, Walley J. Clinical diagnosis of smear-negative pulmonary tuberculosis in low-income countries: the current evidence. Lancet Infect Dis 2003;3:288-96.

3 Raqib R, Rahman J, Kamaluddin AK, et al. Rapid diagnosis of active tuberculosis by detecting antibodies from lymphocyte secretions. J Infect Dis 2003;188:364-70.

4 Raqib R, Kamal SM, Rahman MJ, et al. Use of antibodies in lymphocyte secretions for detection of subclinical tuberculosis infection in asymptomatic contacts. Clin Diagn Lab Immunol 2004;11:1022-7.

5 Rekha RS, Kamal SM, Andersen P, et al. Validation of the ALS assay in adult patients with culture confirmed pulmonary tuberculosis. PLoS One 2011;6:e16425.

6 Raqib R, Mondal D, Karim MA, et al. Detection of antibodies secreted from circulating Mycobacterium tuberculosis-specific plasma cells in the diagnosis of pediatric tuberculosis. Clin Vaccine Immunol 2009;16:521-7.

7 Thomas T, Brighenti S, Andersson J, et al. A new potential biomarker for childhood tuberculosis. Thorax 2011:66:727-9.

8 Chang HS, Sack DA. Development of a novel in vitro assay (ALS assay) for evaluation of vaccine-induced antibody secretion from circulating mucosal lymphocytes. Clin Diagn Lab Immunol 2001;8:482-8.

9 Kirkpatrick BD, Bentley MD, Thern AM, et al. Comparison of the antibodies in lymphocyte supernatant and antibody-secreting cell assays for measuring intestinal mucosal immune response to a novel oral typhoid vaccine (M01ZH09). Clin Diagn Lab Immunol 2005:12:1127-9.

10 Qadri F, Ahmed T, Ahmed F, et al. Mucosal and systemic immune responses in patients with diarrhea due to CS6-expressing enterotoxigenic Escherichia coli. Infect Immun 2007;75:2269-74.

11 Chan ED, Heifets L, Iseman MD. Immunologic diagnosis of tuberculosis: a review. Tuber Lung Dis 2000;80:131-40.

12 Steingart KR, Henry M, Laal S, et al. Commercial serological antibody detection tests for the diagnosis of pulmonary tuberculosis: a systematic review. PLoS Med 2007:4:e202.

13 Odendahl M, Mei $\mathrm{H}$, Hoyer BF, et al. Generation of migratory antigen-specific plasma blasts and mobilization of resident plasma cells in a secondary immune response. Blood 2005;105:1614-21.

14 Jacobi AM, Mei H, Hoyer BF, et al. HLA-DRhigh/CD27high plasmablasts indicate active disease in patients with systemic lupus erythematosus. Ann Rheum Dis 2010;69:305-8

15 Mei HE, Yoshida T, Sime W, et al. Blood-borne human plasma cells in steady state are derived from mucosal immune responses. Blood 2009;113:2461-9.

16 Doria-Rose NA, Klein RM, Manion MM, et al. Frequency and phenotype of human immunodeficiency virus envelope-specific $B$ cells from patients with broadly cross-neutralizing antibodies. J Virol 2009;83:188-99.

17 Dutton RW, Bradley LM, Swain SL. T cell memory. Annu Rev Immunol 1998;16:201-23.

18 Mack U, Migliori GB, Sester M, et al. LTBI: latent tuberculosis infection or lasting immune responses to M. tuberculosis? A TBNET consensus statement. Eur Respir J 2009:33:956-73.

19 Jafari C, Thijsen S, Sotgiu G, et al. Bronchoalveolar lavage enzyme-linked immunospot for a rapid diagnosis of tuberculosis: a Tuberculosis Network European Trials group study. Am J Respir Crit Care Med 2009;180:666-73. 
20 Shah NS, Moodley P, Babaria P, et al. Rapid diagnosis of tuberculosis and multidrug resistance by the microscopic-observation drug-susceptibility assay. Am J Respir Crit Care Med 2011;183:1427-33.

21 Ha DT, Lan NT, Kiet VS, et al. Diagnosis of pulmonary tuberculosis in HIV-positive patients by microscopic observation drug susceptibility assay. J Clin Microbiol 2010;48:4573-9.

22 Slifka MK, Antia R, Whitmire JK, et al. Humoral immunity due to long-lived plasma cells. Immunity 1998;8:363-72.

23 Manz RA, Lohning M, Cassese $G$, et al. Survival of long-lived plasma cells is independent of antigen. Int Immunol 1998;10:1703-11.

24 Sousa AO, Henry S, Maroja FM, et al. IgG subclass distribution of antibody responses to protein and polysaccharide mycobacterial antigens in leprosy and tuberculosis patients. Clin Exp Immunol 1998;111:48-55.

25 Da Costa CT, Khanolkar-Young S, Elliott AM, et al. Immunoglobulin G subclass responses to mycobacterial lipoarabinomannan in HIV-infected and non-infected patients with tuberculosis. Clin Exp Immunol 1993;91:25-9.

26 Verma RK, Jain A. Antibodies to mycobacterial antigens for diagnosis of tuberculosis. FEMS Immunol Med Microbiol 2007;51:453-61.

27 Bothamley GH. Serological diagnosis of tuberculosis. Eur Respir J Supp/ 1995;20:676s-88s.

28 Maekura R, Okuda Y, Nakagawa $M$, et al. Clinical evaluation of anti-tuberculous glycolipid immunoglobulin $\mathrm{G}$ antibody assay for rapid serodiagnosis of pulmonary tuberculosis. J Clin Microbiol 2001;39:3603-8.
29 Leidl L, Mayanja-Kizza H, Sotgiu G, et al. Relationship of immunodiagnostic assays for tuberculosis and numbers of circulating CD4+ T-cells in HIV infection. Eur Respir J 2010;35:619-26.

30 Ferrara G, Losi M, Meacci M, et al. Routine hospital use of a new commercial whole blood interferon-gamma assay for the diagnosis of tuberculosis infection. Am J Respir Crit Care Med 2005;172:631-5.

31 Huebner RE, Schein MF, Hall CA, et al. Delayed-type hypersensitivity anergy in human immunodeficiency virus-infected persons screened for infection with Mycobacterium tuberculosis. Clin Infect Dis 1994;19:26-32.

32 Veldsman C, Kock MM, Rossouw T, et al. QuantiFERON-TB GOLD ELISA assay for the detection of Mycobacterium tuberculosis-specific antigens in blood specimens of HIV-positive patients in a high-burden country. FEMS Immunol Med Microbiol 2009;57:269-73.

33 Titanji K, De Milito A, Cagigi A, et al. Loss of memory B cells impairs maintenance of long-term serologic memory during HIV-1 infection. Blood 2006;108:1580-7.

34 De Milito A, Nilsson A, Titanji K, et al. Mechanisms of hypergammaglobulinemia and impaired antigen-specific humoral immunity in HIV-1 infection. Blood 2004;103:2180-6.

35 Li Y, Zeng Q, Ellis MK, et al. CD4+ T-cell counts, CD4+/CD8+ T-cell count ratios, and antibody levels in migrant fishermen infected with Schistosoma japonicum in the Dongting Lake, China. Am J Trop Med Hyg 2006;75: 910-13. 